\title{
Towards affordable Uncertainty Quantification in the Simulation of Turbulent Spray Combustion via Surrogate Modeling
}

\author{
B. Enderle*, B. Rauch, F. Grimm ${ }^{\dagger}$ and M. Aigner ${ }^{\dagger}$ \\ German Aerospace Center (DLR) \\ Institute of Combustion Technology \\ Pfaffenwaldring 38-40, 70569 Stuttgart, Germany
}

\begin{abstract}
Non-intrusive uncertainty quantification methods mostly rely on space filling sampling of the simulation model over the parameter space of input uncertainties. However, this direct sampling approach is costly for complex applications such as reacting multiphase flows or spray combustion due to the high computational demand. A common strategy is therefore the approximation of the high fidelity simulation via a suitable surrogate model. Thus, only sparse sampling of the actual simulation model over the considered parameter space is required for the generation of surrogate model training data. This paper examines the feasibility of different methods for the construction of surrogate models for spray combustion applications with a high dimension of input parameters and multiple output Quantities of Interest (QoI).

Therefore, the Delft Spray in Hot Coflow flame is investigated by means of reacting multiphase simulations. In this testcase, main input uncertainties arise from the specification of a spray boundary condition replacing the need to model the primary atomization of the fuel injector. Radial profiles of temperature over the flame zone are considered as QoIs. Different sampling methods such as Central Composite Design (CCD), Latin Hypercube Sampling (LHS) and a low discrepancy Sobol sequence are used for the derivation of surrogate model training data. A second order polynomial approximation, Multivariate Adaptive Regression Splines (MARS) and Gaussian Process based Kriging (GP) are selected as surrogate models. The effect of combined sampling strategy and choice of surrogate model on surrogate model quality is determined through local and global cross validation as well as hold out validation against additional simulation data. An efficient and reliable approach is found in the combination of Sobol sequence sampling plan and GP surrogate model. Finally, the application of the constructed surrogate models in an uncertainty quantification of the testcase considered is demonstrated.
\end{abstract}

\section{Nomenclature}

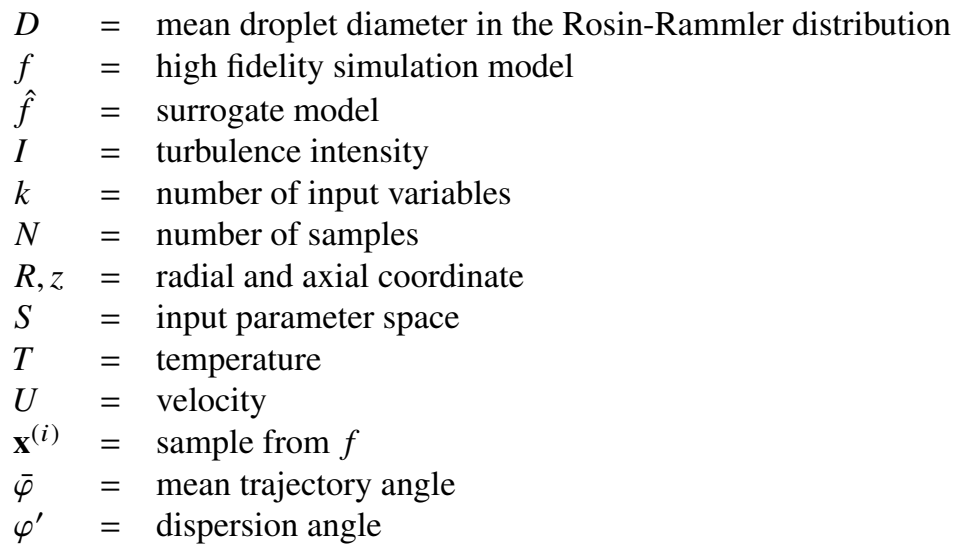

* Research Associate, DLR, Institute of Combustion Technology, Stuttgart, Germany

${ }^{\dagger}$ Senior Scientist, DLR, Institute of Combustion Technology, Stuttgart, Germany

Professor, DLR, Director of the Institute of Combustion Technology, Stuttgart, Germany 


\section{Introduction}

$\mathbf{I}^{\mathrm{N}}$ recent years, Computational Fluid Dynamics (CFD) has emerged as an important tool in both research and lengineering for the analysis of a variety of problems including non-reacting fluid flow, gaseous and liquid combustion or heat transfer [1]. Among them, turbulent spray combustion is still considered one of the most challenging problems due to elaborate modeling requirements for liquid phase dynamics, fuel evaporation, turbulent mixing and reaction in the gas phase [2]. Moreover, the strong non-linear coupling of these sub-processes introduces a distinct sensitivity of the simulations regarding boundary conditions which gives rise to uncertainties in the simulation outcome. In contrast, spray combustion CFD models currently do not yield statistical measures of these uncertainties as a result of their deterministic formulation. However, in the effort to further qualify numerical simulations as a design tool in engineering, uncertainties should be quantified and reported to support reliability and risk-informed decision making.

A common approach for the quantification of uncertainties in an arbitrary simulation model is the non-intrusive propagation of all known input and modeling uncertainties through the simulation model by space filling sampling of the input parameter space $S$ which is constructed from the vector of uncertain parameters $x$ [3]. Yet, due to the high computational demand this direct sampling approach is costly for complex applications such as turbulent spray combustion. One way to overcome this limitation is replacing the high fidelity simulation model $f$ by a suitable surrogate model $\hat{f}$ taking into account minor approximation errors. Then, space filling sampling is conducted on the cheap-to-evaluate surrogate model. The general surrogate model framework is displayed in Fig. 1] For the construction of $\hat{f}$ for a Quantity of Interest (QoI) $y, N$ discrete samples $\left\{\boldsymbol{x}^{(i)} \rightarrow y^{i}=f\left(\boldsymbol{x}^{(i)}\right) \mid i=1, \ldots, N\right\}$ are taken from $f$ via a sparse sampling plan of the input parameter space $S$. The surrogate model is then fitted to the discrete samples (training data) of the simulation model. A variety of surrogate models are found in the literature and choosing the appropriate one depends on the desired accuracy and available training data (e.g. noisy data) [4, 5].

While surrogate model based uncertainty quantification has been demonstrated for gaseous combustion simulations [6-8], only few studies are available on reacting multiphase flow. Gel et al. [9] used a Multivariate Adaptive Regression Spline (MARS) surrogate model to study uncertainties in the simulation of a fluidized bed gasifier. In a further work on the same testcase both nonparametric surfaces such as Gaussian process model and parametric polynomial regression were tested [10]. Consequently, the aim of this paper is examining the feasibility of different methods for the construction of surrogate models for spray combustion applications with a high dimension of input parameters and multiple output QoIs.

The paper is structured as follows. Section $\Pi$ III introduces a testcase for spray combustion based on a laboratory scale ethanol flame. Details on the numerical simulation model are given in section [V] Input uncertainties of the simulation model are then characterized in Section $\nabla$ followed by an overview on different sampling plans for the acquisition of training data and the considered surrogate models in Section VI and VII respectively. Finally, Section VIII reports the application of the aforementioned methods to the testcase and demonstrates the use of the constructed surrogate model for non-intrusive uncertainty quantification.

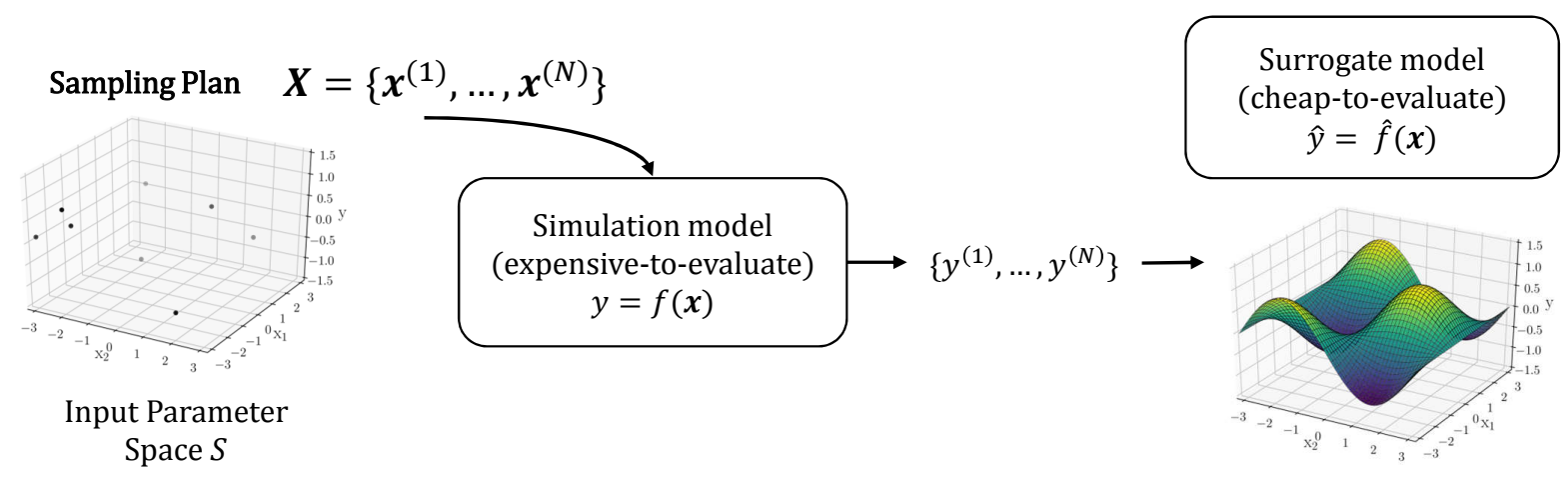

Fig. 1 Surrogate model framework following [5]. 


\section{Test Case: Delft Spray in Hot Coflow (DSHC) Flame}

The Delft Spray in Hot Coflow (DSHC) Flame [11] is selected as a test case for spray combustion due to its simple geometry and extensive available experimental data. As depicted in Fig. 2, the burner consists of a cylindrical hot coflow generator fed by the lean combustion of Dutch Natural Gas (DNG) to increase the airflow temperature prior to the primary combustion zone. A commercial hollow cone pressure swirl atomizer (Delavan WDA 0.5 GPH) is installed in the center of the coflow generator. The atomizer forms a fine spray of ethanol droplets which quickly evaporate and feed a stable lifted of flame above the burner.

Radial profiles of gas phase temperature and droplet sizes over the reaction zone are available from Coherent Anti-Stokes Raman Scattering (CARS) and Phase Doppler Anemometry (PDA) measurements, respectively [11]. Measurement data was collected at different heights above the atomizer $(z=\{15,20,30,40,50,60\} \mathrm{mm})$. Reported operating conditions for both coflow generator and atomizer are listed in Tab 1.

\section{Table 1 Reported operating conditions.}

\begin{tabular}{cccccccc}
\hline Case & $T_{c f}[\mathrm{~K}]$ & $U_{c f}\left[\mathrm{~m} \mathrm{~s}^{-1}\right]$ & $I_{c f}[\%]$ & $m_{\text {liq. }}\left[\mathrm{kg} \mathrm{h}^{-1}\right]$ & $p_{\text {inj }}[\mathrm{bar}]$ & $T_{\text {liq. }}[\mathrm{K}]$ & $\varphi\left[{ }^{\circ}\right]$ \\
\hline $\mathbf{H}-\mathrm{II}$ & 1400 & 2.5 & 2.0 & 1.46 & 11.5 & 301 & 30 \\
\hline
\end{tabular}

\section{Numerical Simulation}

\section{A. RANS Simulation Model}

In a prior study [12] Large Eddy Simulations (LES) of the DSHC flame demonstrated excellent agreement with the experimental data. However, the present study is based on training data from simplified Reynols Averaged Navier Stokes Simulations (RANS) due to the significant reduction in computational expense which allows for a multitude of simulations compared to LES.

As in [12], simulations are carried out with a DLR (German Aerospace Center) inhouse CFD code for reacting multiphase flows, named THETA/SPRAYSIM. The unstructured finite-volume gas phase solver THETA [13] (Turbulent Heat Release Extension for the TAU Code) is optimized with regard to low Mach number combustion problems typical for gas turbine combustors. Steady state Reynolds Averaged Navier Stokes simulations involving a $k-\varepsilon$ turbulence model and a Finite Rate Chemistry combustion model with detailed chemical kinetics [14] are used for the gas phase. For the simulation of the liquid phase, THETA is coupled with the DLR inhouse code SPRAYSIM [15],

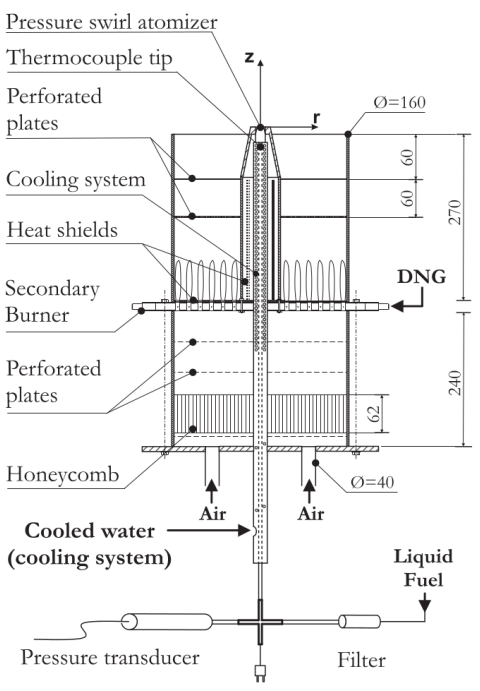

Fig. 2 Schematic of the DSHC burner [11].
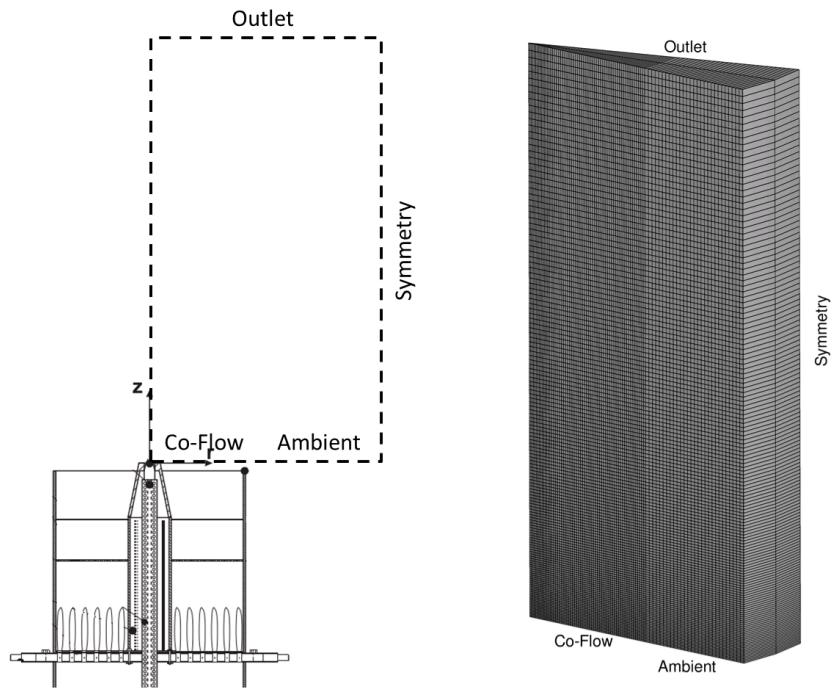

Fig. 3 Sketch of the computational domain and grid. 
where transport and evaporation of liquid droplets is tracked in a Lagrangian framework. Evaporation is addressed using the vaporization model of Abramzon and Sirignano [16], turbulent droplet dispersion is accounted for by a variance of the Gosman-Ioannides dispersion model [17].

A detail of the computational domain is given in Fig. 3. Only the region above the atomizer is considered in the simulations, while the properties of the coflow after secondary combustion such as temperature, velocity and species composition are prescribed as a boundary condition based on the experimental characterization. For further simplification solely a $20^{\circ}$ wedge rather than a full cylinder is simulated which is justified by the symmetry of the considered problem. An ambient inflow with $U=0.1 \mathrm{~m} \mathrm{~s}^{-1}$ surrounds the co-flow. At the outlet, zero static pressure is set. The fully structured grid as shown in Fig. 3 consists of approximately $40 \cdot 10^{3}$ elements with strong clustering in the liquid injection region. $190 \mathrm{CPU}$-hours are required for a single simulation with this setup.

Spatial convergence of the simulation is examined using the Grid Convergence Index (GCI) method as proposed by Roache [18]. The flow field is computed on three different grids, maximum temperature $T_{\max }$ in the computational domain is considered as a solution functional. Tab. 2 2 summarizes results from the grid study with a GCI of $0.12 \%$ for the reference grid, i.e. the grid used in this work. Based on this study it is concluded that the spatial resolution of the reference grid is sufficient for the RANS simulation model.

Fig. 44provides a comparison of the LES results from [12] and the used RANS simulation with experimental data for radial profiles of gas phase temperature at different heights above the atomizer. Only minor deviation between the two simulation approaches is visible except for $R \leq \pm 5 \mathrm{~mm}$, where the RANS systematically overpredicts the temperature over all axial positions. However, it is concluded that the proposed simplified setup is suitable for the following study.
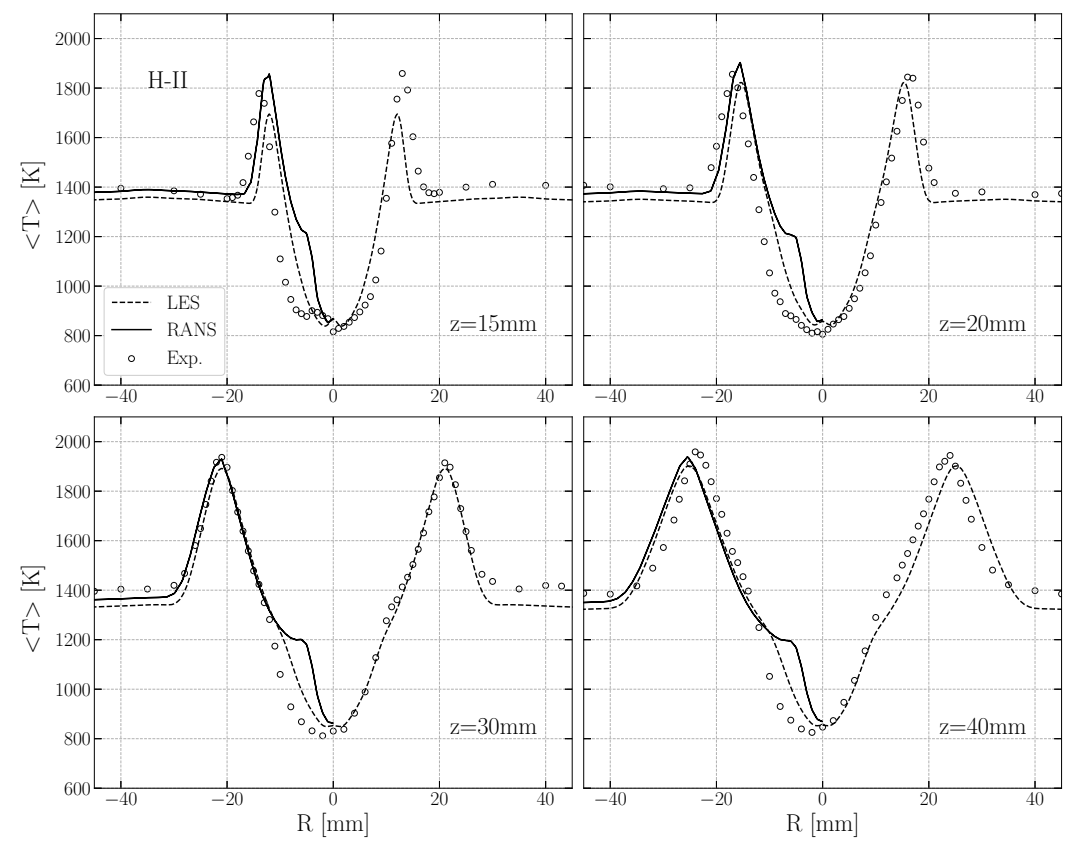

Fig. 4 Radial profiles of gas phase temperature at different heights above the atomizer from LES and RANS.

Table 2 Grid convergence study based on maximum temperature $T_{\max }$.

\begin{tabular}{lcccc}
\hline Grid & & $n_{p n t}[-]$ & $T_{\max }[\mathrm{K}]$ & $G C I[\%]$ \\
\hline Fine & $f_{1}$ & 81106 & 1942.22 & - \\
Reference & $f_{2}$ & 40015 & 1941.29 & 0.12 \\
Coarse & $f_{3}$ & 19688 & 1941.29 & - \\
Extrapolation & $f_{\infty}$ & - & 1942.23 & - \\
\hline
\end{tabular}




\section{B. Spray Boundary Condition}

In the simulations, primary atomization of the liquid fuel is not calculated due to the complex physical phenomena and resulting computational costs. Therefore, a spray boundary condition must be specified describing the dispersed spray of droplets after primary atomization. This is achieved via an injection disk with diameter $d_{i n}=1 \mathrm{~mm}$ at a distance of $z=1 \mathrm{~mm}$ from the actual atomizer where liquid droplets are issued into the computational domain. An illustration of the geometric quantities at the injection disk is given in Fig. 5 . The spray half cone is defined by the mean droplet trajectory angle $\bar{\varphi}$ and a dispersion angle $\varphi^{\prime}$. All droplets are injected with a velocity magnitude of $U$. The droplet size spectrum in the polydisperse spray is modeled by a Rosin-Rammler distribution,

$$
Q(D)=1-\exp (X / D)^{q} \quad \text { with } \quad X=\operatorname{SMD}\left[\Gamma\left(1-\frac{1}{q}\right)\right],
$$

and $q=3$.

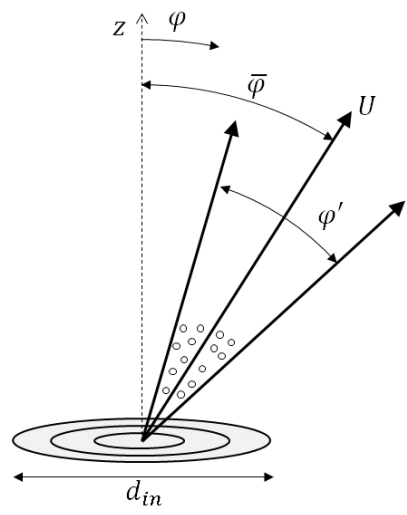

Fig. 5 Depiction of spray boundary condition and input parameters at the injection plane.

\section{Non-Deterministic Approach}

Since gas phase temperature data is one of the major output quantities of a spray combustion simulation, the radial temperature profiles at different heights above the atomizer as shown in Fig. 4 are considered as QoI for the following non-deterministic simulation study.

\section{A. Modeling of the Input Uncertainties}

For the characterization of the uncertainties of the given simulation model a systematic approach as proposed by Roy and Oberkampf [19] is followed which can be subdivided into two fundamental steps:

\section{Identification of all relevant sources of uncertainty}

From a theoretical point of view, sources of uncertainties of a given simulation model consist of three major categories, namely numerical uncertainties, model form and input uncertainties. Numerical uncertainties take into account errors arising from the approximation of the differential equation-based model, such as discretization error, iterative convergence error or round-off error. Model form uncertainties stem from the process of abstraction and formulation of the mathematical models and can be categorized into omission, aggregation and substitution type [3]. Finally, input uncertainties include parameters used in the simulation model or its sub-models as well as data describing the surrounding of the system, i.e. boundary conditions.

However, the following analysis will be focused on input uncertainties only as they play a major role in the simulation of turbulent spray combustion as a result of the complexity of the required boundary conditions and the high sensitivity of the simulation outcome on input variation. Moreover, experimental data characterizing the dispersed spray after primary atomization is subject to high measurement uncertainties. Consequently, spray boundary conditions commonly require calibration against experimental results. For example, Ma et al. pointed out that using the droplet 
injection velocity based on the experimental data of the DSHC flame, the downstream velocity will be significantly overpredicted in the simulation [20].

As the inflow boundary condition of the gas phase is fully characterized by measurement data, special emphasis will be put on the spray boundary condition as defined in section [IV.B From an a priori Moris One at A Time (MOAT) [21] screening study, four parameters of the spray boundary condition where found to be most influential on the Quantity of Interest (QoI), namely the mean droplet diameter in the Rosin-Rammler distribution $D$, the liquid droplet injection velocity $U_{l i q .}$, the mean droplet trajectory angle $\bar{\varphi}$ and the dispersion angle $\varphi^{\prime}$.

\section{Characterization of the selected sources of uncertainty}

In this step a mathematical structure defining each source of uncertainty and numerical values describing this structure must be assigned. Due to the aforementioned necessity to calibrate the spray boundary condition, different values of the four considered uncertain inputs $\left(D, U_{\text {liq. }}, \varphi, \varphi^{\prime}\right)$ are found in the literature on simulations of the DSHC flame [20, 22,-24] which primarily emerge from the different simulation models used. We construct the uncertain input parameter space from the minimum and maximum values of the input quantities found in the literature. Since no further information is available, all four input quantities are treated as purely epistemic interval-valued uncertainties bounded by the minimum and maximums as summarized in Tab. 3 In addition, data from [12] is given as a reference being the optimum configuration for the used simulation framework. Due to the epistemic nature of the uncertainties, no mathematical structure of the input probability density function (PDF) is defined.

Table 3 Minimum and maximum bounds of the epistemic uncertain inputs.

\begin{tabular}{lcccc}
\hline & $D[\mu \mathrm{m}]$ & $U_{\text {liq. }}\left[\mathrm{m} \mathrm{s}^{-1}\right]$ & $\bar{\varphi}\left[{ }^{\circ}\right]$ & $\varphi^{\prime}\left[{ }^{\circ}\right]$ \\
\hline Minimum & 40 & 27.3 & 30 & 6 \\
Maximum & 45 & 35.7 & 40 & 20 \\
\hline Reference & 42.5 & 35.7 & 30 & 20 \\
\hline
\end{tabular}

\section{Sampling of Training Data}

In this section, sampling plans $\boldsymbol{X}=\left\{\boldsymbol{x}^{(1)}, \boldsymbol{x}^{(2)}, \ldots, \boldsymbol{x}^{(n)}\right\}$ for the acquisition of surrogate model training data $\left\{\left(\boldsymbol{x}^{(1)}, \boldsymbol{y}^{(1)}\right),\left(\boldsymbol{x}^{(2)}, \boldsymbol{y}^{(2)}\right), \ldots,\left(\boldsymbol{x}^{(n)}, \boldsymbol{y}^{(n)}\right)\right\}$ from the simulation model are discussed. From each major classes of sampling plans, i.e. classical design, random design and quasi-random design, one example is considered. Special emphasis is put on the suitability of the sampling plan for the later construction of surrogate models.

\section{A. Classical Design: Central Composite Design}

A central composite design (CCD) contains an embedded factorial or fractional factorial design augmented with a group of center and axial points (also known as star points). The star point consists of new extrema for each design variable. As the CCD was originally developed for planning physical experiments it is sometimes referred to as a "classical design of experiments" in contrast to the "modern" types specifically constructed for computer experiments. Note that the number of sample points $N$ specified in a CCD is a function of the number of variables $k$ in the problem:

$$
N=1+2 k+2^{k} .
$$

\section{B. Random Design: Latin Hypercube Sampling}

Random designs aim on generating sampling points that have an equally likely probability to appear in any sample. In order to avoid clustering tendencies of crude Monte Carlo methods, stratified sampling techniques such as Latin Hypercube Sampling (LHS) divide the range of input variables into segments of equal probability. When sampling a function of $k$ variables, the range of each variable is divided into $m$ equally probable intervals. $N=m$ sampling points are then placed to satisfy the Latin Hypercube requirements [25], forcing the number of division to be equal for each variable. 

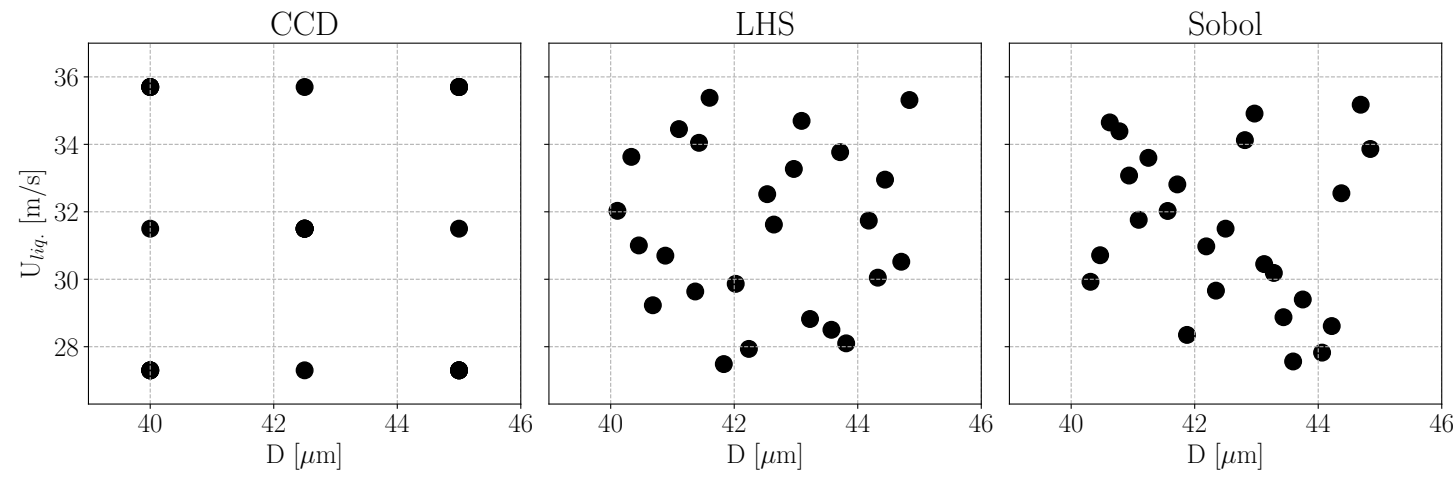

Fig. 6 Sampling from different sampling plans in the $D-U_{l i q}$. parameter space $(N=25)$.

\section{Quasi-random Design: Sobol Sequence}

Also called low discrepancy sequences generators, quasi random design approaches seek to bridge the gap between the flexibility of random sampling techniques and the advantages of grid designs [5]. Various quasi-random sampling methods have been constructed [26] in an attempt to minimize the spatial discrepancy of the sampling, i.e. how the spread of the sampling points deviate from a uniform spread over the design space. Amongst them, a low discrepancy sequence as proposed by Sobol [27] is selected. Sobol sequences have the favorable property that for any $N$ and $k>1$, the sequence for $N-1$ and $k$ is a subset of the sequence for $N$ and $k$, making them easy to extend by adding points to an existing sequence [4]. This is in contrast to LHS plans, where the entire design must be recomputed if an LHS plan containing more samplings is required.

Taking the CCD as a reference, $N=25$ samples resulting from $k=4$ are taken from each sampling plan. Twodimensional projections of exemplary samples from the three sampling plans with $N=25$ are plotted in Fig. 6. As all input uncertainties are characterized as purely epistemic, a uniform level of surrogate model accuracy and therefore a uniform spread of the sampling points throughout the parameter space is required. Hence, spatial discrepancy of the aforementioned sampling plans is evaluated using the centered L2-discrepancy norm [28]:

$$
\begin{aligned}
C^{2}\left(X_{k}^{N}\right)=\left(\frac{13}{12}\right)^{k} & -\frac{2}{N} \sum_{i=1}^{N} \prod_{n=1}^{k}\left(1+\frac{1}{2}\left|x_{n}^{(i)}-0.5\right|-\frac{1}{2}\left|x_{n}^{(i)}-0.5\right|^{2}\right) \\
& +\frac{1}{N^{2}} \sum_{i, j=1}^{N} \prod_{n=1}^{k}\left(1+\frac{1}{2}\left|x_{n}^{(i)}-0.5\right|+\frac{1}{2}\left|x_{n}^{(j)}-0.5\right|-\frac{1}{2}\left|x_{n}^{(i)}-x_{n}^{(j)}\right|\right) .
\end{aligned}
$$

It can be interpreted as the difference between the distribution of samples in the sampling plan and the uniform distribution with $C^{2} \rightarrow 0$ for a uniform distribution. Tab. 4 summarizes $C^{2}$ results from sampling plans considered. As expected, the random and quasi-random designs return a notably lower spatial discrepancy than the CCD.

Table 4 Centered L2-discrepancy for different sampling plans and sizes.

\begin{tabular}{cccc}
\hline & Central Composite Design & Latin Hypercube Sampling & Sobol Sequence \\
\hline$C^{2}\left(X_{4}^{25}\right)$ & 0.48 & 0.08 & 0.09 \\
\hline
\end{tabular}

\section{Surrogate Modeling}

Based on the training data, surrogate models are constructed using Sandias toolbox for validation, verification and uncertainty quantification DAKOTA 6.4 [29]. At each axial measurement position $z=\{15,20,30,40,50,60\} \mathrm{mm}, 50$ equidistant radial positions within $R \leq 50 \mathrm{~mm}$ are defined as observation points. Consequently, 300 surrogate models $\hat{T}^{x, R}$ have to be constructed for the evaluation of the temperature profiles.

Three different types of data fit surrogate models are considered: 


\section{A. Second Order Polynomial}

Polynomial regressions are one of the most common and cheapest surrogates to fit data as a low-order polynomial model is often an accurate approximation to true data trends over a small portion of the parameter space. In addition, the least-square procedure in the determination of polynomial coefficients provides a surface fit that smooths out noise in the data [5]. Although in general, approximation of the modeled function $f(\boldsymbol{x})$ can be achieved with polynomials $\hat{f}(\boldsymbol{x})$ of any order, a second order polynomial

$$
\hat{f}(\boldsymbol{x}) \approx c_{0}+\sum_{i=1}^{n} c_{i} x_{i}+\sum_{i=1}^{n} \sum_{j \geq i}^{n} c_{i j} x_{i} x_{j}
$$

is utilized in this study. The $x_{i}, x_{j}$ terms are the components of the $n=k$-dimensional design parameter values, the $c_{0}, c_{i}, c_{j}, c_{i j}$ terms are the polynomial coefficients and $n=k$ is the number of design parameters. For the second order polynomial, at least

$$
n_{c}=\frac{(k+1)(k+2)}{2}
$$

training data samples must be available to fully determine the linear system. In DAKOTA, a least-squares approach involving a singular value decomposition numerical method is applied to solve the linear system [29].

\section{B. Multivariate Adaptive Regression Splines (MARS)}

MARS is a nonparametric, piecewise polynomial splines-based surface fitting method that can represent complex multimodal trends [30]. The general form of the MARS model is based on the following expression:

$$
\hat{f}(\boldsymbol{x}) \approx \sum_{m=1}^{M} a_{m} B_{m}(\boldsymbol{x}),
$$

where the $a_{m}$ are the coefficients of the truncated power basis functions $B_{m}$, and $M$ is the number of basis functions. MARS builds multiple linear regression models across the range of sampling data by partitioning the data and running a linear regression model on each of the partitions before joining them together to a $C^{2}$-continuous surface model [29]. For the construction of MARS surrogate models, DAKOTA utilizes the MARS3.5 package developed at Stanford University.

Although MARS models are satisfactory enough to deal with moderately large data sets, they are susceptible to overfitting and sometimes erroneous when dealing with gappy data [5].

\section{Gaussian Process based Regression}

The basic idea of Gaussian Process based Regression stems from the assumption that each training data point is correlated with its neighbors and every regression point can be seen as a multivariate Gaussian distribution. The surrogate model $\hat{f}(\boldsymbol{x})$ is then built on realizations of a random Gaussian Process (GP). The form of the GP based Regression model reads

$$
\hat{f}(\boldsymbol{x}) \approx \boldsymbol{g}(\boldsymbol{x})^{T} \boldsymbol{\beta}+\boldsymbol{r}(\boldsymbol{x})^{T} \boldsymbol{R}^{-1}(\boldsymbol{f}-\boldsymbol{G} \boldsymbol{\beta})
$$

where $\boldsymbol{x}$ is the current point in the $N$-dimensional parameter space; $\boldsymbol{g}(\boldsymbol{x})$ is the vector of trend basis functions evaluated at $\boldsymbol{x} ; \boldsymbol{\beta}$ is a vector containing the generalized least squares estimates of the trend basis function coefficients; $\boldsymbol{r}(\boldsymbol{x})$ is the correlation vector of terms between $\boldsymbol{x}$ and the training points; $\boldsymbol{R}$ is the correlation matrix for all of the training points; $\boldsymbol{f}$ is the vector of response values; and $\boldsymbol{G}$ is the matrix containing the trend basis functions evaluated at all training points. The terms in the correlation vector and matrix are computed using a Gaussian correlation function and are dependent on an $N$-dimensional vector of correlation parameters, $\boldsymbol{\theta}=\left\{\theta_{1}, \ldots, \theta_{N}\right\}^{T}$. DAKOTA determines the value of $\boldsymbol{\theta}$ using a Maximum Likelihood Estimator procedure.

\section{Results and Discussion}

\section{A. RANS based Training Data}

A comparison of radial temperature profiles from RANS simulations is given in Fig. 77. Nine samples from the LHS training dataset are drawn, each corresponding to a realization in the parameter space. The equivalent sample 

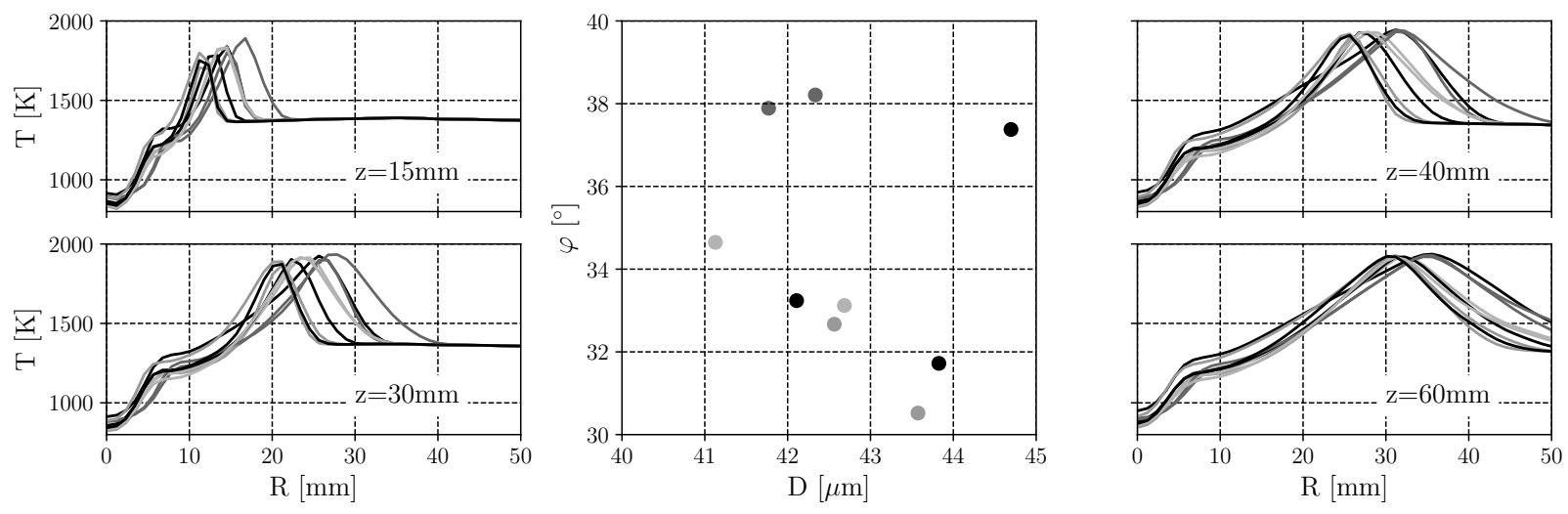

Fig. 7 Temperature profiles at different training data points in the parameter space from RANS simulation.

position in the $D-\varphi$ subspace is indicated by colored dots in the central plot of Fig. 77. From these temperature profiles it is already evident that the considered variation in input parameters results in significant variations of the temperature distribution over the computational domain. Especially the radial position of the peak temperature, which is connected to the reaction zone of the flame, varies over a range of $\Delta r=10 \mathrm{~mm}$ at all axial positions. In contrast, the hot gas temperature from the coflow outside of the reaction zone $(R>5 \mathrm{~mm}$ at $z=15 \mathrm{~mm})$ is not affected by the varying droplet properties.

Although main characteristic of the problem considered are already observable in the training dataset, for a rigorous uncertainty quantification, space filling sampling of parameter space is required which can only be achieved using surrogate models.

\section{B. Construction of Surrogate Models}

Based on the training data from the different sampling plans surrogate models are constructed by DAKOTA using the methods discussed in the previous sections. Tab. 5 summarizes the combinations of sampling plans and surrogate models investigated in the following.

\section{Examination of Surrogate Model Quality}

As the constructed surrogate models will be used instead of the actual RANS simulation for detailed uncertainty analysis of the testcase, surrogate model reliability and quality must be assessed. The fact that a surrogate model will always be an approximation of the true simulation model gives rise to a surrogate model prediction error

$$
\varepsilon_{S M}=\mathrm{QoI}_{S i m} .-\mathrm{QoI}_{S M},
$$

which is the difference between the prediction of a quantity of interest from the simulation and the corresponding surrogate model [4]. Two validation and testing techniques, namely cross validation and holdout validation, are used to estimate $\varepsilon_{S M}$ and benchmark the cases from Tab. 5 regarding surrogate model quality.

Table 5 Considered combinations of sampling plans (Plan) and surrogate models (SM).

\begin{tabular}{|c|c|c|c|}
\hline Plan $S M$ & GP & MARS & Quadratic \\
\hline CCD & $\checkmark$ & $\checkmark$ & $\checkmark$ \\
\hline LHS & $\checkmark$ & $\checkmark$ & $\checkmark$ \\
\hline Sobol & $\checkmark$ & $\checkmark$ & $\checkmark$ \\
\hline
\end{tabular}



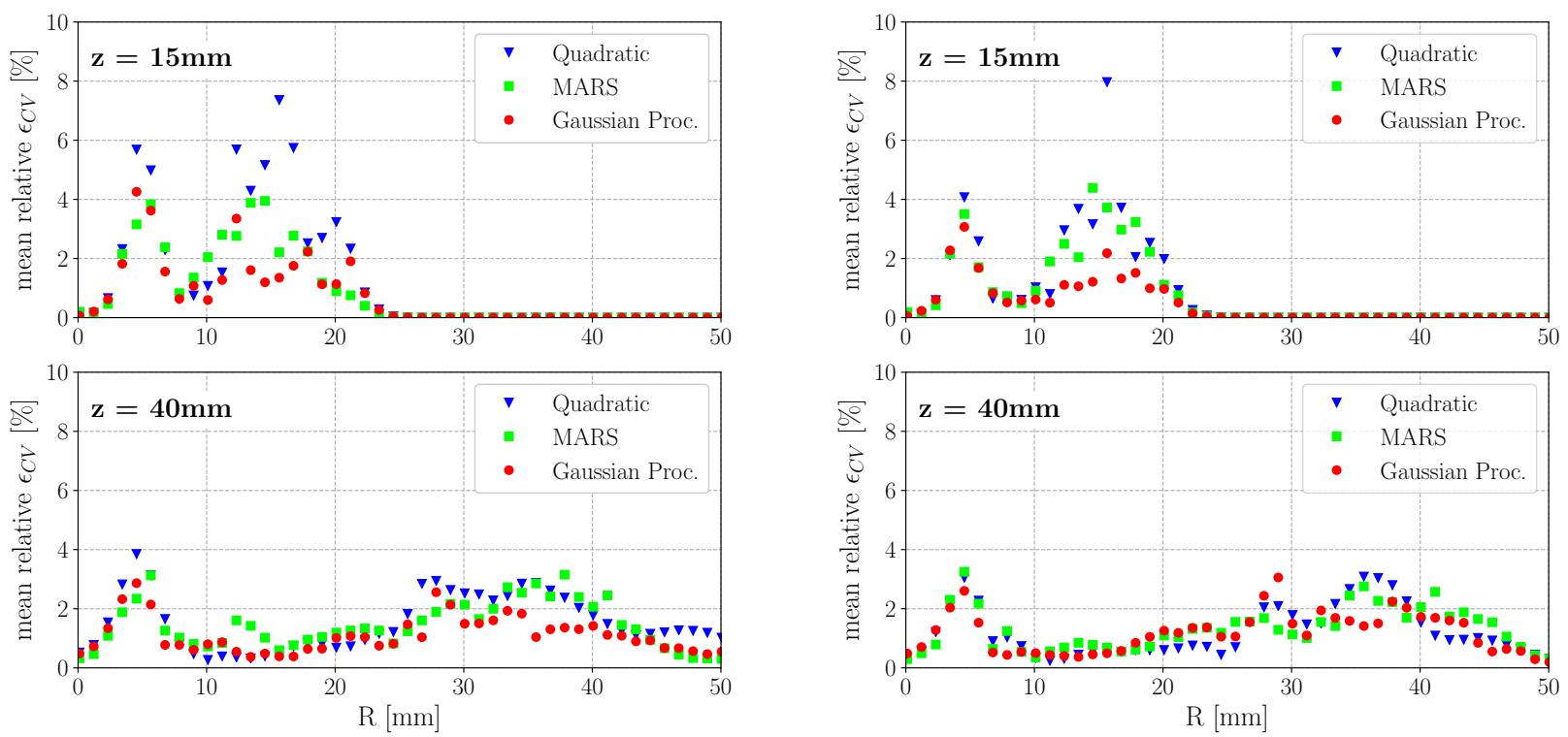

(a) LHS

(b) Sobol

Fig. 8 Radial profiles of mean relative cross-validation error $\varepsilon_{C V}$ for different surrogate model and sampling plan combinations

\section{Model validation: Cross Validation}

In cross-validation the set of training data is split into $q$ equal subsets followed by the construction of the surrogate model on $q-1$ subsets while keeping the remaining subset for model testing. This procedure is subsequently repeated for all subsets. Consequently, the mean cross-validation error reads

$$
\varepsilon_{C V}=\frac{1}{n} \sum_{i=1}^{q} \varepsilon_{S M, i}
$$

In this study, the training dataset is split into $q=N$ subsets for a leave-one-out cross-validation of the local surrogate models $\hat{T}^{x, R}$.

Radial profiles of mean cross-validation errors at two axial positions are given in Fig. 8 The left column presents results using the LHS sampling plan in combination with the different surrogate models: At $z=15 \mathrm{~mm}$ two distinct peaks of $\varepsilon_{C V}$ are present at $R=5 \mathrm{~mm}$ and $R=15 \mathrm{~mm}$. Outside of the flame zone $(R>25 \mathrm{~mm})$ the error drops to zero. Highest local errors result from the Quadratic Surrogate Model with $\max \left(\varepsilon_{C V}\right)=7.5 \%$. In contrast, the maximum error from the Gaussian Process stays below 4.5\%. At the further downstream position at $z=40 \mathrm{~mm}$, the maximum error decreases for all surrogate models. The same tendencies are visible in the error profiles from Sobol sampling based surrogate models in Fig. 8 (b). However, maximum local error for the MARS and Gaussian Process are slightly decreased at $z=15 \mathrm{~mm}$.

Table 6 Input parameters for additional Holdout Validation test data.

\begin{tabular}{ccccc}
\hline Case & $D[\mu \mathrm{m}]$ & $U_{\text {liq. }}\left[\mathrm{m} \mathrm{s}^{-1}\right]$ & $\bar{\varphi}\left[{ }^{\circ}\right]$ & $\varphi^{\prime}\left[{ }^{\circ}\right]$ \\
\hline HV1 & 41.25 & 29.4 & 32.5 & 9.5 \\
HV2 & 43.75 & 33.6 & 32.5 & 16.5 \\
HV3 & 41.25 & 33.6 & 37.5 & 16.5 \\
HV4 & 43.75 & 29.4 & 37.5 & 9.5 \\
\hline
\end{tabular}




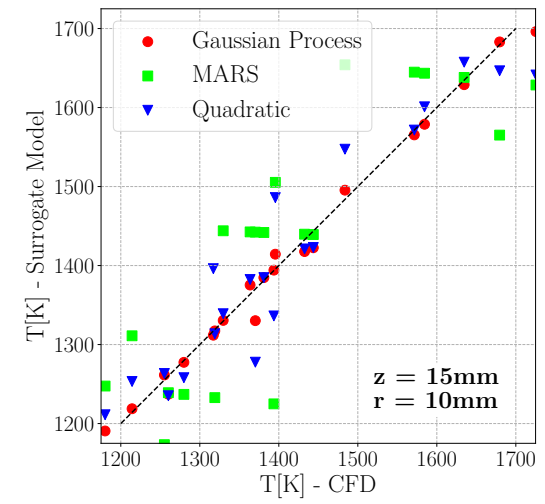

(a) $\mathrm{CCD}$

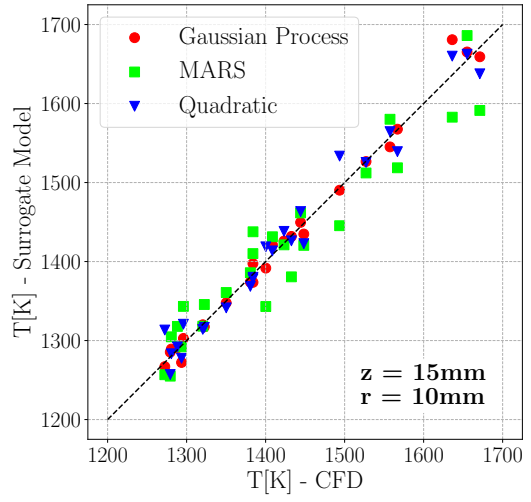

(b) LHS

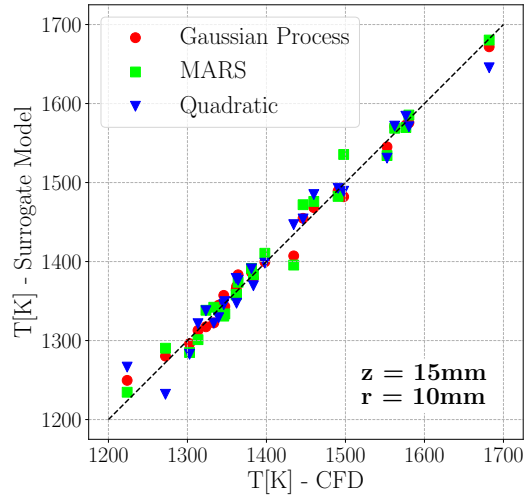

(c) Sobol

Fig. 9 Local cross validation plots at $z=15 \mathrm{~mm}, r=10 \mathrm{~mm}$ for different sampling plans.

As an example of local cross-validation data of a single QoI, the position at $z=15 \mathrm{~mm}$ and $R=10 \mathrm{~mm}$ is selected from the plot in Fig. 8 In the experiments this position is connected to the highest temperature gradient in the flame zone and therefore subject to high uncertainties in the simulation. Results from the 25 leave-one-out runs are plotted in Fig. 9 by means of cross-validation plots. In the ideal case all points would line up along the dashed diagonal. Contrarily, the deviation from the diagonal indicates the single run cross validation error. The mean cross-validation error from Eq. 8 increases as more points deviate from the diagonal. Over the three sampling plans in Fig. 9 (a)-(c) the deviation for the MARS model decreased when moving from a CCD to the Sobol plan while the Gaussian Process error is not affected by the change in sampling plan. A slight improvement is also visible for the Quadratic model. In conclusion, the Sobol sampling plans improves the surrogate model quality at this position.

\section{Model Testing: Holdout Validation}

In addition to the 25 RANS model evaluations from the sampling plans, four independent points in the parameter space of the problem considered are simulated and kept aside for model testing. Consequently, the constructed surrogate models are tested against new data and the predictive capability can be visualized. Tab. 6 summarizes input parameters for the additional Holdout Validation simulations.

A comparison of surrogate model prediction and CFD simulation for case HV2 at two different axial positions is given in Fig. 10. Each column comprises results from surrogate models based on the three different sampling plans. All combinations are able to predict the temperature profiles at both axial positions with minor deviation. However, in the CCD based results in Fig. 10 (a) the MARS model underestimates the temperature distribution in the peak region at $z=40 \mathrm{~mm}$ and overestimates the transition region between flame and coflow at $z=40 \mathrm{~mm}$. This deviation diminishes when moving to an LHS or Sobol sampling plan in Fig. 10 (b) and (c). Thus, the MARS model profits from the reduced spatial discrepancy of the LHS and Sobol sampling plan compared to the CCD. Comparing the three surrogate model types, the Gaussian Process captures the simulation profiles with best accuracy over all three sampling

Table 7 Mean Holdout Validation error $\mu\left|\varepsilon_{H V}\right|$ for the considered combinations of sampling plans (Plan) and surrogate models (SM).

\begin{tabular}{|c|c|c|c|}
\hline Plan & GP & MARS & Quadratic \\
\hline $\mathrm{CCD}$ & $1.38 \%$ & $2.28 \%$ & $1.52 \%$ \\
\hline LHS & $0.68 \%$ & $0.84 \%$ & $0.95 \%$ \\
\hline Sobol & $0.59 \%$ & $0.75 \%$ & $0.69 \%$ \\
\hline
\end{tabular}



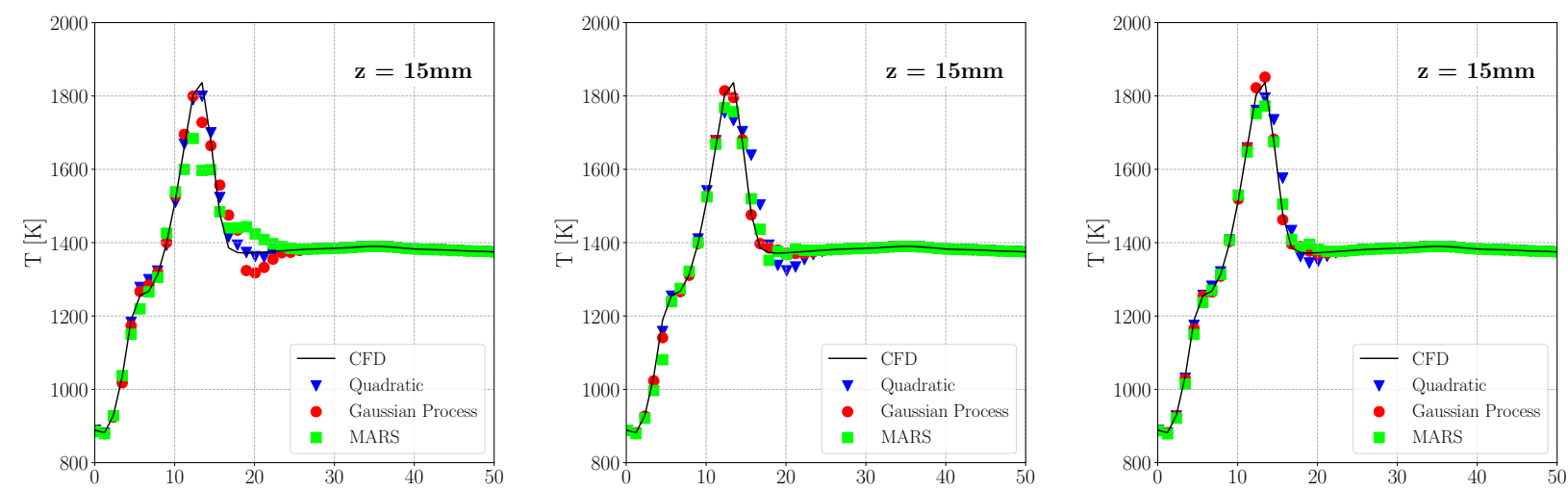

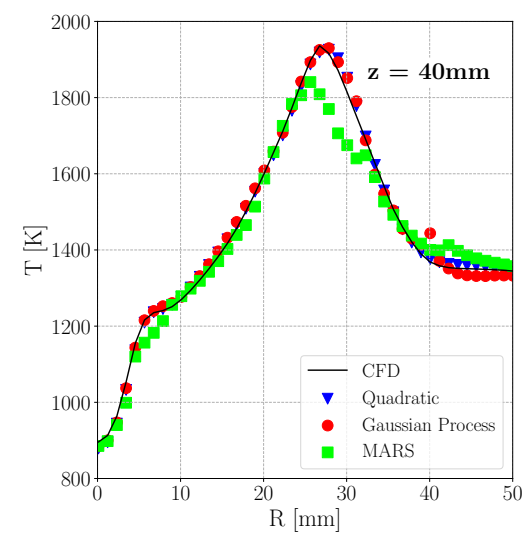

(a) $\mathrm{CCD}$

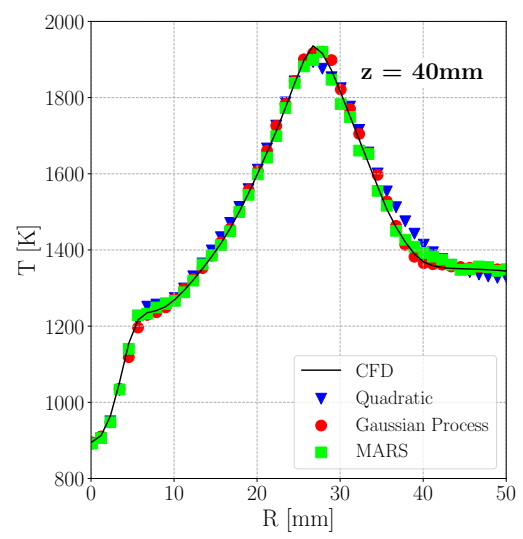

(b) LHS

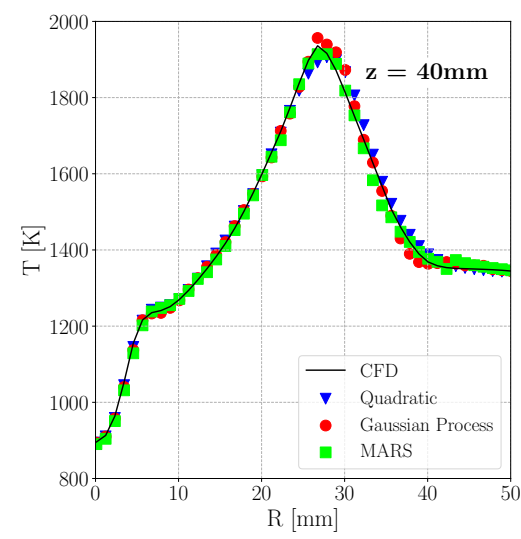

(c) Sobol

Fig. 10 Surrogate model predictions and CFD simulation results for Holdout Validation case HV2.

plans. Although not shown here for brevity, the discussed features are found in all four Holdout Validation cases. For a further quantification of Holdout Validation results, the relative error $\varepsilon_{C V}$ between surrogate model prediction $\hat{T}$ and holdout data $T$ is computed at each axial and radial position $z, R$ for the four cases from Tab 6 .

$$
\varepsilon_{H V}^{x, R}=\frac{T^{x, R}-\hat{T}^{x, R}}{T^{x, R}} .
$$

Histograms of the resulting 1200 error samples for each case are plotted in Fig.11 The CCD sampling plan returns the broadest spectrum of errors up to $\pm 10 \%$ whereas LHS and Sobol stay below $\pm 5 \%$. Furthermore, the frequency around $\varepsilon_{H V}=0 \%$ increases for the latter two indicating enhanced prediction quality. To compare the different surrogate models, mean Holdout Validation error $\mu\left|\varepsilon_{H V}\right|$ is provided. The Gaussian Process based models reveal the lowest mean error over all sampling plans with a minimum of $0.59 \%$ in combination with Sobol sampling. As already identified in Cross Validation, the LHS and Sobol plans reduce the error of MARS surrogate models compared to CCD sampling.

\section{Application to Uncertainty Quantification}

Based on the previous surrogate model quality assessment, the Gaussian Process in combination with the Sobol training data is used to showcase a non-deterministic simulation of the considered spray combustion problem. Due to the purely epistemic nature of the input uncertainties the Probability Bounds Analysis framework (PBA) [3, 31] is utilized. $10^{5}$ samples of the input parameter space as defined in Tab. 3 are drawn from Latin Hypercube Sampling. For each sampling point the surrogate models are evaluated allowing for an economical propagation of input uncertainties. The surrogate based computation of all $10^{5}$ samples is achieved in a few minutes which is orders of magnitude faster than the same analysis on the original simulation model.

Fig. 12 shows the surrogate based non-deterministic simulation results for the DSHC H-II case in comparison with the deterministic simulation results from the RANS model and the experimental data. As a consequence of the 


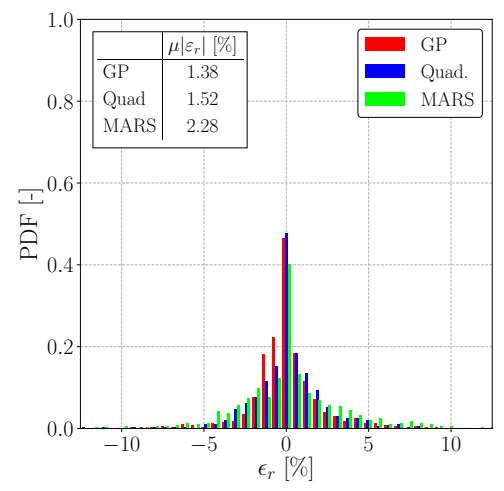

(a) $\mathrm{CCD}$

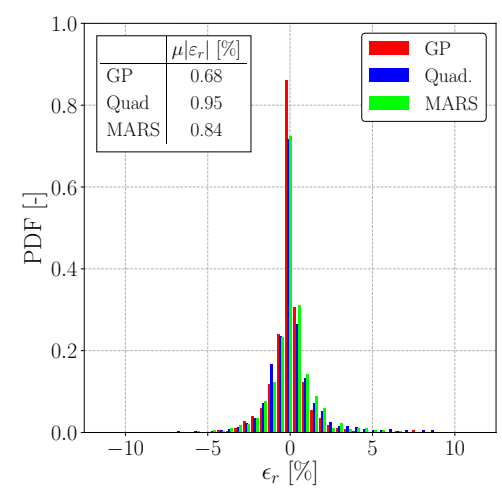

(b) LHS

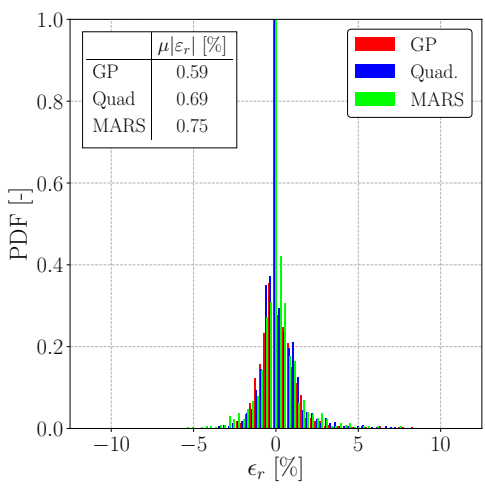

(c) Sobol

Fig. 11 Histograms of Holdout Validation error $\varepsilon_{H V}$ from cases HV1 - HV4.

PBA framework and the epistemic input all realizations in the non-deterministic simulation are given a probability of unity. Therefore, the grey area represents the uncertainty region of the simulation model defined by the given input uncertainties. Minimum and maximum of the local QoI bound the uncertainty region. Highest uncertainties exist in the region of peak temperature at all axial positions indicating that the spray input parameters significantly influence the position of the flame. Over all radial positions in the flame region an uncertainty level between $100 \mathrm{~K}$ and $1400 \mathrm{~K}$ is found.

The fact that some portions of the experimental data are not covered by the uncertainty region gives rise to the assumption that there exist either additional uncertainties in the simulation model which are not covered in this analysis or a general modeling error. Furthermore, uncertainties in the experimental data are not represented.

It is evident that the non-deterministic simulation approach now offers a more detailed insight into the considered problem than the deterministic approach: The visibility of all uncertainties in the simulation results allows for a risk-informed decision about the predictive capabilities of the simulation model, i.e. a quantitative measure for the precision of the simulation model under given uncertainties.
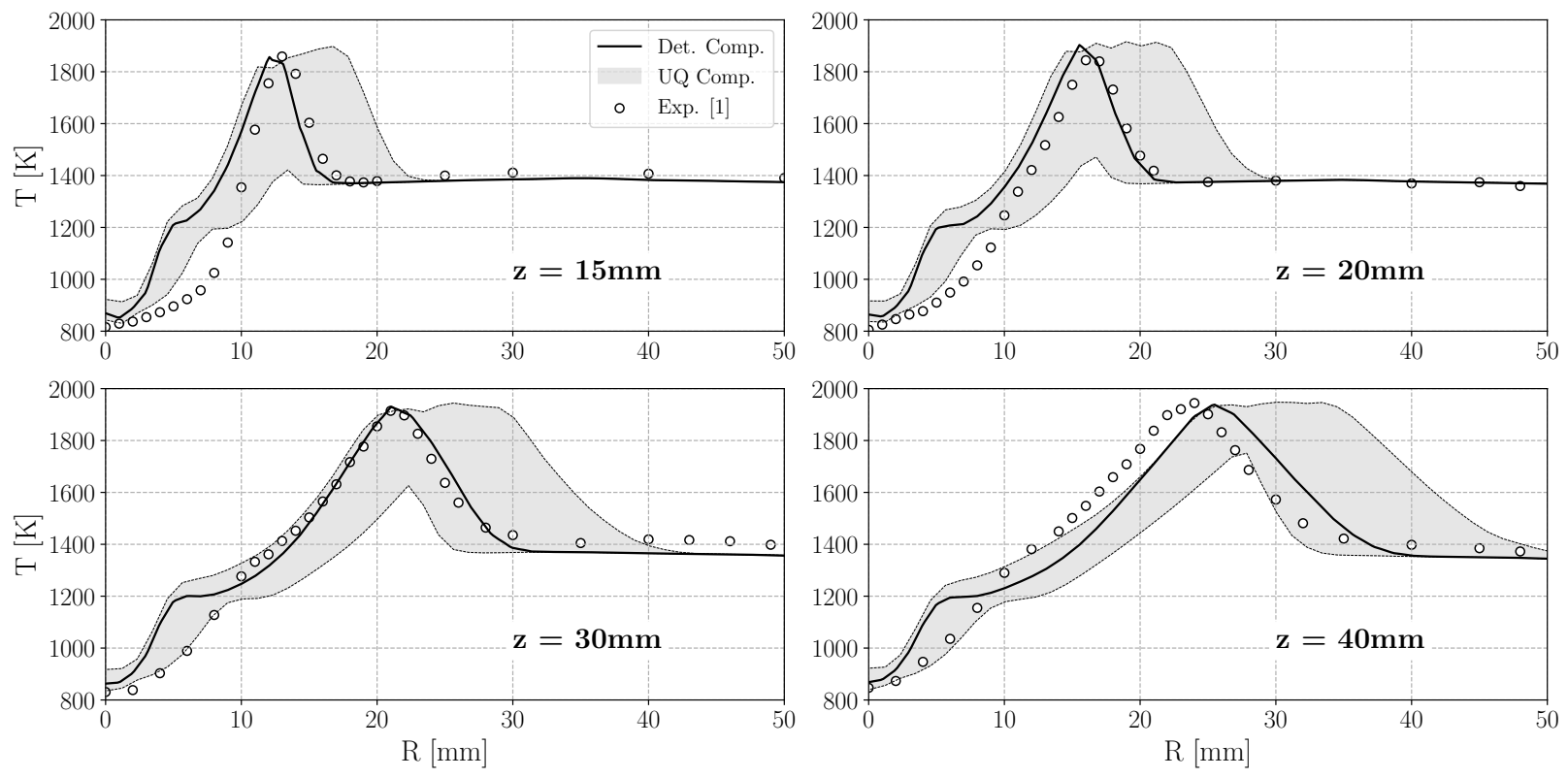

Fig. 12 Surrogate based non-deterministic simulation results of the DSHC H-II case. 


\section{Conclusion and Future Work}

Although deterministic computational simulation methods for turbulent spray combustion are able to reproduce experimental data in well defined testcases, the high modeling demand and the complexity of boundary conditions potentially cause uncertainties in the simulation results. In the effort to further qualify numerical simulations as a design tool in engineering, these uncertainties can only be quantified through transitioning to non-deterministic simulation approaches.

A strategy for affordable uncertainty quantification in complex turbulent spray combustion simulations was successfully demonstrated in the present paper. A workflow consisting of the acquisition of training data from high fidelity simulations, construction of surrogate models and space filling sampling of the surrogate model was applied to a spray combustion simulation for the first time. Special emphasis was put on the assessment of surrogate model quality. The combination of Sobol sequence based sampling and a Gaussian Process surrogate model was identified as the most reliable approximation method. Non-deterministic simulations for temperature profiles of the testcase revealed an extensive uncertainty region around the existing deterministic results and highlighted the importance of rigorous uncertainty quantification for this application.

Applying the proposed workflow to engineering design problems such as the preliminary design of a gas turbine combustor would aid in identifying performance critical regions of the design under the given input uncertainties. Based on these findings, a decision about investing resources in reducing the input uncertainties to safely meet performance targets such as emissions or efficiency could be made.

In a following study, the non-deterministic simulations should be extended to additional Quantities of Interest from the dispersed phase such as evaporated mass fraction or profiles of droplet diameter in order to get insight into the coupled effects between gaseous and dispersed phase caused by the input uncertainties. The cheap-to-evaluate approximation via surrogate modeling also enables variance based sensitivity analysis such as Sobol' Indices [32] which require a high amount of sampling statistics. This method could aid in understanding the connection between input parameters and output variance.

\section{Acknowledgments}

The authors would like to thank Prof. D. M. Roekarts from TU Delft for providing the experimental database on the DSHC flame.

\section{References}

[1] Colombo, E., Inzoli, F., and Mereu, R., "A methodology for qualifying industrial CFD: The Q3 approach and the role of a protocol,” Computers $\mathcal{E}$ Fluids, Vol. 54, 2012, pp. 56-66.

[2] Jenny, P., Roekaerts, D., and Beishuizen, N., "Modeling of turbulent dilute spray combustion," Progress in Energy and Combustion Science, Vol. 38, No. 6, 2012, pp. 846-887.

[3] Oberkampf, W. L., and Roy, C. J., Verification and validation in scientific computing, Cambridge University Press, 2010.

[4] Forrester, A., Keane, A., et al., Engineering design via surrogate modelling: a practical guide, John Wiley \& Sons, 2008.

[5] Yondo, R., Andrés, E., and Valero, E., "A review on design of experiments and surrogate models in aircraft real-time and many-query aerodynamic analyses," Progress in Aerospace Sciences, 2017.

[6] Khalil, M., Lacaze, G., Oefelein, J. C., and Najm, H. N., "Uncertainty quantification in LES of a turbulent bluff-body stabilized flame," Proceedings of the Combustion Institute, Vol. 35, No. 2, 2015, pp. 1147-1156.

[7] Johnson, R., Wu, H., and Ihme, M., "A general probabilistic approach for the quantitative assessment of LES combustion models," Combustion and Flame, Vol. 183, 2017, pp. 88-101.

[8] Mueller, M. E., Iaccarino, G., and Pitsch, H., "Chemical kinetic uncertainty quantification for large eddy simulation of turbulent nonpremixed combustion,” Proceedings of the Combustion Institute, Vol. 34, No. 1, 2013, pp. 1299-1306.

[9] Gel, A., Garg, R., Tong, C., Shahnam, M., and Guenther, C., "Applying uncertainty quantification to multiphase flow computational fluid dynamics," Powder technology, Vol. 242, 2013, pp. $27-39$.

[10] Gel, A., Li, T., Gopalan, B., Shahnam, M., and Syamlal, M., "Validation and uncertainty quantification of a multiphase computational fluid dynamics model," Industrial E Engineering Chemistry Research, Vol. 52, No. 33, 2013 , pp. $11424-11435$. 
[11] Rodrigues, H. C., Tummers, M. J., van Veen, E. H., and Roekaerts, D. J., "Spray flame structure in conventional and hot-diluted combustion regime," Combustion and Flame, Vol. 162, No. 3, 2015, pp. 759-773.

[12] Enderle, B., Rauch, B., Grimm, F., and Aigner, M., "Large Eddy Simulation of Turbulent Ethanol Spray Flames under MILD conditions using a Finite Rate Chemistry Combustion Model," Proceedings of ICLASS 2018, 14th Triennial International Conference on Liquid Atomization and Spray Systems, 2018.

[13] Di Domenico, M., Gerlinger, P., and Noll, B., "Numerical simulations of confined, turbulent, lean, premixed flames using a detailed chemistry combustion model," ASME 2011 Turbo Expo: Turbine Technical Conference and Exposition, American Society of Mechanical Engineers, 2011, pp. 519-530.

[14] Röhl, O., and Peters, N., "A reduced mechanism for ethanol oxidation," 4th European Combustion Meeting (ECM 2009), Vienna, Austria, April, 2009, pp. 14-17.

[15] Eckel, G., Rachner, M., Le Clercq, P., and Aigner, M., "Semi-Empiricalmpirical Model for the unsteady Shear Breakup of liquid Jets in Cross-Flow," Atomization and Sprays, Vol. 26, No. 7, 2016.

[16] Abramzon, B., and Sirignano, W., "Droplet vaporization model for spray combustion calculations," International journal of heat and mass transfer, Vol. 32, No. 9, 1989, pp. 1605-1618.

[17] Gosman, A., and Loannides, E., “Aspects of computer simulation of liquid-fueled combustors,” Journal of energy, Vol. 7, No. 6, 1983, pp. 482-490.

[18] Roache, P. J., "Perspective: a method for uniform reporting of grid refinement studies," Journal of Fluids Engineering, Vol. 116, No. 3, 1994, pp. 405-413.

[19] Roy, C. J., and Oberkampf, W. L., "A comprehensive framework for verification, validation, and uncertainty quantification in scientific computing," Computer methods in applied mechanics and engineering, Vol. 200, No. 25, 2011, pp. 2131-2144.

[20] Ma, L., and Roekaerts, D., "Modeling of spray jet flame under MILD condition with non-adiabatic FGM and a new conditional droplet injection model," Combustion and Flame, Vol. 165, 2016, pp. 402-423.

[21] Morris, M. D., "Factorial sampling plans for preliminary computational experiments," Technometrics, Vol. 33, No. 2, 1991, pp. $161-174$.

[22] Gallot-Lavallée, S., Jones, W., and Marquis, A., "Large Eddy Simulation of an ethanol spray flame under MILD combustion with the stochastic fields method," Proceedings of the Combustion Institute, 2016.

[23] Ma, L., Zhu, S., Tummers, M., Van Der Meer, T., and Roekaerts, D., "Numerical investigation of ethanol spray-in-hot-coflow flame using steady flamelet model," Eighth Mediterranean Combustion Symposium, 2013, pp. 1-12.

[24] Jamali, S. H., "Computational Modeling of Turbulent Ethanol Spray Flames in a Hot Diluted Coflow," Ph.D. thesis, TU Delft, Delft University of Technology, 2014.

[25] Santner, T. J., Williams, B. J., and Notz, W. I., The design and analysis of computer experiments, Springer Science \& Business Media, 2013.

[26] Niederreiter, H., Random number generation and quasi-Monte Carlo methods, Vol. 63, Siam, 1992.

[27] Sobol, I. M., Asotsky, D., Kreinin, A., and Kucherenko, S., "Construction and Comparison of High-Dimensional Sobol'Generators," Wilmott, Vol. 2011, No. 56, 2011, pp. 64-79.

[28] Hickernell, F., "A generalized discrepancy and quadrature error bound," Mathematics of Computation of the American Mathematical Society, Vol. 67, No. 221, 1998, pp. 299-322.

[29] et. al, B. M. A., "Dakota, A Multilevel Parallel Object-Oriented Framework for Design Optimization, Parameter Estimation, Uncertainty Quantification, and Sensitivity Analysis: Version 6.4 User's Manual,” Sandia Technical Report SAND2014-4633, 2016.

[30] Friedman, J. H., "Multivariate adaptive regression splines," The annals of statistics, 1991, pp. 1-67.

[31] Ferson, S., and Ginzburg, L. R., "Different methods are needed to propagate ignorance and variability," Reliability Engineering $\mathcal{E}$ System Safety, Vol. 54, No. 2-3, 1996, pp. 133-144.

[32] Sobol, I. M., "Sensitivity estimates for nonlinear mathematical models," Mathematical modelling and computational experiments, Vol. 1, No. 4, 1993, pp. 407-414. 